\title{
A Novel Energy-Efficient Plasma Chemical Process for the Destruction of Volatile Toxic Compounds
}

\author{
Oak Ridge National Laboratory \\ September 1997 \\ Progress Report
}

Principal Investigator

Lal A. Pinnaduwage

(423) 574-6540 (Phone)

(423) 574-6210 (Fax)

1lp@ornl.gov

Oak Ridge National Laboratory

P.O. Box 2008

Oak Ridge, TN 37831-6122

Co-Investigator

Cheng-Yu Lin Ma

(423) 576-0991 (Phone)

(423) 576-7956 (Fax)

xlc@ornl.gov

Oak Ridge National Laboratory

P.O. Box 2008

Oak Ridge, TN 37831-6120

\section{Contributors}

D. L. McCorkle, ORNL

W. Ding, ORNL 


\section{Research Objective}

The objective of this research program is to develop new plasma chemical processes for the destruction of volatile toxic compounds (VTCs) in contaminated air streams where the contamination levels are below a few percent. We plan to exploit the large cross sections associated with dissociative electron attachment to highly excited molecular states. Such highly excited states are to be populated in glow discharges via excitation transfer from high-lying, metastable states of rare gases. Basic knowledge of the excitation transfer processes and the electron attachment processes are crucial to the development of the proposed techniques, and these processes will be studied in detail.

\section{Research Statement}

We are pursuing two techniques for the destruction of VTCs: (1) a pulsed glow discharge scheme and (2) a plasma mixing scheme. In the pulsed discharge apparatus we are subjecting dilute mixtures to pulsed discharges and measuring the contaminant levels at the reactor output using a newly developed analytical technique. We are conducting somewhat more fundamental studies with a plasma mixing apparatus to gain a better understanding of the basic chemical processes involved.

\section{Research Progress}

\section{The Pulsed Glow Discharge Scheme}

We have assembled the complete apparatus consisting of the reaction chamber and the analytical instruments to analyze the reactor output. The gas mixture to be processed flows through the glow discharge region and can be diverted to a sampling loop for analysis. The gas flow system is complete with flow and pressure monitors and an oil-free pump. The sampling loop is connected to a gas chromatograph (GC) so that the VTC concentration in the discharge output can be quantified. The sampling loop is also connected to a mass spectrometer (MS) so that the discharge byproducts can be identified. A gas handling system was assembled to prepare dilute mixtures of VTCs.

Because the reactor is operated at pressures less than 10 torr, conventional analytical techniques could not be used to analyze the reactor output. We devised a system for sampling and preconcentrating VTCs for the subsequent GC analysis. GC analysis was generally used to quantify the remaining VTCs after the destruction by plasma discharge. In some cases, combined GC/MS was employed to identify the destruction products in order to examine the plasma discharge mechanism.

This analytical methodology was validated for the analysis of $400 \mathrm{ppm}$ methylene chloride (in argon) using $400 \mathrm{ppm}$ octane (in argon) as the internal standard (IS). The calibration curve was constructed by plotting the pressure ratios of methylene chloride to IS versus the corresponding peak area ratios. Over a pressure range of 0.1 to 25.0 torr, at least triplicate analyses were carried out for each of the ten data points used to construct the calibration curve. 
The relative standard deviation (RSD) for the entire pressure range varied from 2.7 to $16.2 \%$, with the high RSD values being associated with the VTC pressures closed to the method detection limit (MDL). The MDL was determined to be 0.85 torr based on the procedure described in the Code of Federal Regulations (40 CFR Part 136, Appendix B). The linearity of the calibration curve was also determined, with slope $=4.56$, intercept $=-0.011$, and correlation coefficient $\left(R^{2}\right)=0.996$. The details of this new analytical technique are described in a paper in preparation for submission to the Journal of Chromatography.

Currently, the above described methodology is being used to determine the destruction efficiency of methylene chloride by plasma discharge processes operating either in the pulsed or in the direct current (DC) mode. In these experiments, a glow discharge is maintained between a cylindrical anode and a cylindrical cathode; the pressure in the reactor is in the range of 1-10 torr, and the flow rate is in the range of 250-1000 standard $\mathrm{c}^{3} / \mathrm{min}$.

Our VTC destruction mechanism is primarily based on (1) population of highly excited states of the VTCs via excitation transfer from the long-lived metastable states of rare gases and (2) efficient dissociative electron attachment to those highly excited states of VTCs that lead to the dissociation of the molecule. Subsequent ion-molecule and radical-molecule reactions are expected to enhance the dissociation of VTCs. In the pulsed discharge methods, the main idea is to populate the metastable states of rare gases during the "pulse on" period and for the excitation transfer and the electron attachment to occur in the "pulse off" period. A crude version of this can be obtained by using a DC discharge. Because the gas flows through the discharge, it is automatically subjected to a "discharge on" cycle and a "discharge off" cycle.

Our measurements so far indicate that even in the DC discharge mode, VTC destruction efficiencies obtained are comparable to other conventional plasma remediation techniques including pulsed corona and dielectric barrier discharge methods. However, our technique is quite inexpensive compared to the other techniques as only a DC power supply is needed to maintain the discharge.

In the experiments conducted with a pulsed power supply (pulse rate $\approx 10 \mathrm{kHz}$ ), the destruction efficiencies obtained are much better compared to our DC discharge method or the other existing plasma-based techniques.

The findings of these experiments are to be presented at the 50th Gaseous Electronics Conference to be held in Madison, Wisconsin, in October 1997. We are also preparing a paper to be submitted to the Journal of Applied Physics.

\section{The Plasma Mixing Scheme}

This apparatus was also constructed during the past year. At present it is designed to conduct basic studies on the role of the metastable states of rare gases and to study dissociative electron attachment to highly excited states populated via excitation transfer from the rare-gas metastable states. This is also a gas flow apparatus, but in this case, only the rare gas is subjected to a glow discharge in the "source region." Plasma from the source region then flows into a "target region" into which a molecular gas (target gas) is fed. It is important to note that the target gas is not directly subjected to the discharge. The plasma that is extracted to the target region contains the long-lived metastable states in addition to electrons. Excitation transfer from the metastable states to the target gas occurs in the target region, and subsequently these excited states undergo dissociative electron attachment. Dissociative electron attachment leads to the destruction of the target molecule; furthermore, the negative ions produced by this process undergo subsequent ion- 
molecule reactions that lead to further destructions. The removal of electrons by dissociative electron attachment is monitored using Langmuir probes.

Using this apparatus, we conducted basic studies to verify the role of the metastable states of rare gases: In these studies we confirmed that if the ionization potential (IP) of the target gas lies below the metastable-state energy, then the molecule is ionized. This information has been known for a long time and is referred to as Penning ionization. On the other hand, if the IP of the target gas lies above the metastable-state energy, then population of the highly-excited states of the target gas occurs. Subsequently, these excited states attach electrons dissociatively to produce fragment negative ions. We found concrete evidence for the latter process. This turned out to be a very efficient process; $30 \%$ to $80 \%$ of the electrons extracted to the target region were observed to be converted to negative ions. The findings of these studies are important not only for this project, but also for the plasma community in general. These findings have been submitted for publication in Applied Physics Letters.

\section{Summary of Accomplishments}

- A plasma reactor was constructed to study plasma remediation of VTCs using a novel pulsed glow discharge scheme. This apparatus is equipped with a GC and also a GUMS to analyze the reactor output.

- A new analytical method was developed to analyze the reactor output, which is at a pressure range of $1-10$ torr.

- Measurements conducted with the above apparatus show that the pulsed glow discharge scheme is more energy efficient compared to the existing plasma-based VTC destruction techniques. Even with a DC glow discharge, efficiencies comparable to the existing schemes can be obtained, at a much lower capital cost.

- A plasma mixing apparatus was constructed to conduct basic studies on the plasma-based VTC destruction process.

- Basic information on the role of the rare-gas metastable states and on dissociative electron attachment to highly excited molecules was obtained using the above apparatus.

\section{Publications and Abstracts}

Ma, C. Y., D. L. McCorkle, W. Ding, and L. A. Pinnaduwage. "A Methodology for Direct Sampling of Volatile Organic Compounds Emerging from a Low Pressure, Flow-Through Reaction Cell for Subsequent GC and/or GC/MS Analysis." To be submitted to Journal of

\section{Chromatography.}

McCorkle, D. L., C. Y. Ma, and L. A. Pinnaduwage. "Destruction of $\mathrm{CH}_{2} \mathrm{Cl}_{2}$ Using a Glow Discharge Scheme." Abstract accepted for the 50th Gaseous Electronics Conference, Madison, Wis., October 6-9, 1997. 
Pinnaduwage, L. A., W. Ding, and D. L. McCorkle. "Enhanced Electron Attachment to HighlyExcited Molecules Using a Plasma Mixing Scheme." Submitted to Applied Physics Letters.

Pinnaduwage, L. A., C. Y. Ma, D. L. McCorkle, and W. Ding. "Novel Glow Discharge Based Methods for Plasma Remediation of Volatile Toxic Compounds." To be submitted to Journal of A pplied Physics. 